\title{
Gate Driver Circuits With Discrete Components For GaN-based Multi-Level Multi-Inductor Hybrid Converter
}

This paper was downloaded from TechRxiv (https://www.techrxiv.org).

LICENSE

CC BY-NC-SA 4.0

SUBMISSION DATE / POSTED DATE

02-09-2021 / 09-09-2021

\section{CITATION}

Das, Ratul; Le, Hanh-Phuc (2021): Gate Driver Circuits With Discrete Components For GaN-based MultiLevel Multi-Inductor Hybrid Converter. TechRxiv. Preprint. https://doi.org/10.36227/techrxiv.16557726.v1

DOI

10.36227/techrxiv.16557726.v1 


\title{
Gate Driver Circuits With Discrete Components For GaN-based Multi-Level Multi-Inductor Hybrid Converter
}

\author{
Ratul Das, Student Member, IEEE, and Hanh-Phuc Le, Senior Member, IEEE
}

\begin{abstract}
Gate driver circuits to ensure proper turnon and turn-off for power switches are essential parts of a power converter design. They become even more important for multilevel converters where multiple switches are operated at active voltage domains. Recent favorable use of Gallium-Nitride (GaN) devices for power switches makes gate driving even more challenging as the switch performance and reliability are more sensitive to variations of the gate driving signals and power compared with traditional power MOSFETs. This paper discusses gate driving methods using a multi-level multi-inductor hybrid (MIH) converter as the demonstration prototype to address two key challenges in designing gate drivers: 1) providing level-shifted PWM signals to active voltage domains and 2) powering schemes for gate driver circuits. To solve the first challenge, an optimal use of available half-bridge drivers is devised to eliminate the need for separate signal isolator chips. This method was implemented and verified in a MIH converter prototype for 48-V Point-of-Load (PoL) applications using three different powering schemes for gate drivers, including isolated power modules, regulated supplies from switch blocking voltages, and cascaded bootstrap power rails with regulations. The gate driver techniques and powering schemes are compared experimentally in terms of performance to illustrate their benefits and trade-offs.
\end{abstract}

\section{INTRODUCTION}

$\mathbf{M}$ ultilevel hybrid DC-DC converters have made significant contributions to bolster the recent rapid expansion of power electronics impact. Their successful integration in power supply for high-performance computing, automotive applications, space devices, and domestic appliances, etc., has paved the path for a long-term strong establishment in the field. Particularly, they emerge as the most appealing candidate for non-isolated Point-of-Load (PoL) converters in data centers and telecommunications systems. Recently, a number of different multi-level hybrid converters have been demonstrated for this application where moderate to extreme step-up or step-down conversions are

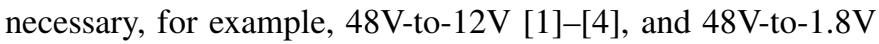
[5]-[14], etc. These converters have been demonstrated with resonant operations for highly-efficient fixed conversion ratios [1]-[3], [5], [6] or regulated output operations with pulse-width modulation (PWM) duty cycle control [4], [7][10], [12]-[14]. With superior performance over conventional Buck converters, these hybrid converters promise significant improvement of the overall system efficiency and power density.

Despite their clear advantages in power conversion, their market penetration is still hindered with the concerns over a number of design challenges, especially gate drivers for a relatively large number of stacked power switches. The designs of these hybrid DC-DC converters share a common characteristic that they utilize more switches and passive components to reduce voltage stress on them individually. The lower voltage stress enables the use of devices with lower voltage ratings and higher density for higher system efficiency and smaller overall space. However, a large number of power switches means a challenge in the increased complexity of gate drivers. To make it worse, many power switches in these multi-level hybrid converters are operated at different voltage domains and shifted levels.

Figure 1 depicts a 2-phase 6-inductor hybrid converter (2P6IHC) [12], [15] as an example of multilevel hybrid converters, utilizing 12 switches, $\mathrm{Sw}_{1-12}$. In this converter, switches $\mathrm{Sw}_{7-12}$ are operated at the ground level, i.e. their Source terminals are connected to the ground, while the other 6 switches, $\mathrm{Sw}_{1-6}$ are stacked on top switch $\mathrm{Sw}_{7}$. Although there are various types of multilevel converters, such as duty cycle controlled flying capacitor multi-level (FCML) hybrid converters [7], [16], [17] and multi-phase multi-inductor hybrid (MIH) converters [10]-[15], [18], and a broad varieties of resonant or hybrid switched [1]-[3], [6], [19], [20], the stacked switch structure similar to $\mathrm{Sw}_{1-7}$ in Fig. 1 is common in these converter families. The ground switches $\mathrm{Sw}_{7-12}$ are relatively straightforward to drive, but it is challenging to drive the six stacked switches $\mathrm{Sw}_{1-6}$ while satisfying reliability and performance across all operating points. The most fundamental stacked switch configuration is a half-bridge. Since gate driver integrated circuits (gate driver ICs) are often intended for only one single switch or two half-bridge switches, there is no single gate driver IC product that can drive a large number of stacked switches in multilevel converters.

This paper presents an optimal use of half-bridge drivers that takes advantage of built-in signal isolators in Section II. In Section III, a review of traditional and discussion of new schemes to generate power rails for the multi-level converter are provided. Section IV presents experimental results and verifications of the circuits presented in Section II and III. The paper is finally summarized and concluded in Section V.

\section{Optimal use OF HALF-BRIDGE DRIVERS}

There are a number of techniques known for powering flying drivers, whose voltage domains are switched in normal operations of converters, some of which will be covered in Section III. However, techniques to level-shift control signals from the ground level to appropriate flying voltage 


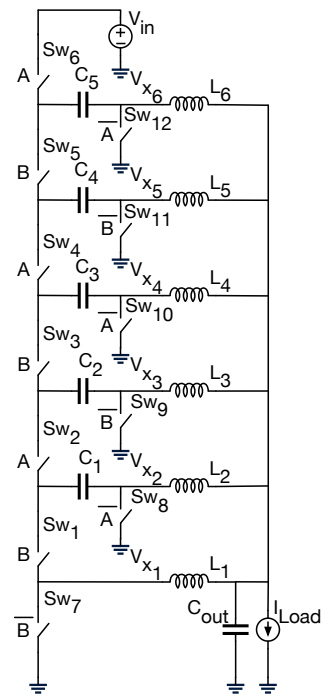

Fig. 1: Multilevel hybrid converter example: 2P6IHC

domains using discrete components can still benefit from further improvements for a smaller discrete component count and space. Commercial signal isolators rely on inductive or capacitive couplings to convey control signals to different voltage levels [21], [22]. Because of the relatively large passives required for isolated coupling and the nature of being discrete components, signal isolator ICs are often areaconsuming and can be even larger than the switches that they are driving. This is a common problem in many power converters that have multiple synchronous power switches such as multi-level converters. In addition, these isolators can also add significant delays to the critical signal path, leading to more complex timing control. It is, therefore, desirable to minimize the number of signal isolator ICs required in converter design.

It is widely known that typical commercial half-bridge driver ICs have a built-in capacitive signal isolator. This halfbridge driver is usually used to drive a half-bridge switch pair where the top driver drives the switch that is stacked immediately on top of the one driven by the bottom driver [1], [17], [23], [24]. However, it is much less recognized that the built-in signal isolator in a half-bridge driver is capable of a large voltage difference and can actually be used in place of a signal isolator. Utilizing this built-in capability can optimize half-bridge gate driver functionality and eliminate the need for all discrete signal isolators in multilevel hybrid converters.

Figure 2 depicts the switched capacitor (SC) part of a Series Capacitor Buck Converter (SCBC) [18] as an example where half-bridge driver ICs and their built-in signal isolators are proposed to be used in a new configuration to remove the need for discrete signal isolators. The power converter architecture has 4 switches in Fig. $3 \mathrm{a}$, two of them, $\mathrm{Sw}_{1}$ and $\mathrm{Sw}_{2}$ are stacked on a ground-level switch $\mathrm{Sw}_{3}$. Switch $\mathrm{Sw}_{4}$ is at the ground level and operated in a complementary phase to $\mathrm{Sw}_{2}$. Flying capacitor $\mathrm{C}_{\mathrm{F} 1}$ connects switch $\mathrm{Sw}_{4}$ to $\mathrm{Sw}_{1}, \mathrm{Sw}_{2}$ and the rest of the circuit.

In this power converter, directly stacked switches $\mathrm{Sw}_{1}$ and $\mathrm{Sw}_{3}$ can be driven by a half-bridge driver IC. As switches $\mathrm{Sw}_{2}$ and $\mathrm{Sw}_{4}$ do not share a common switching node as in usual

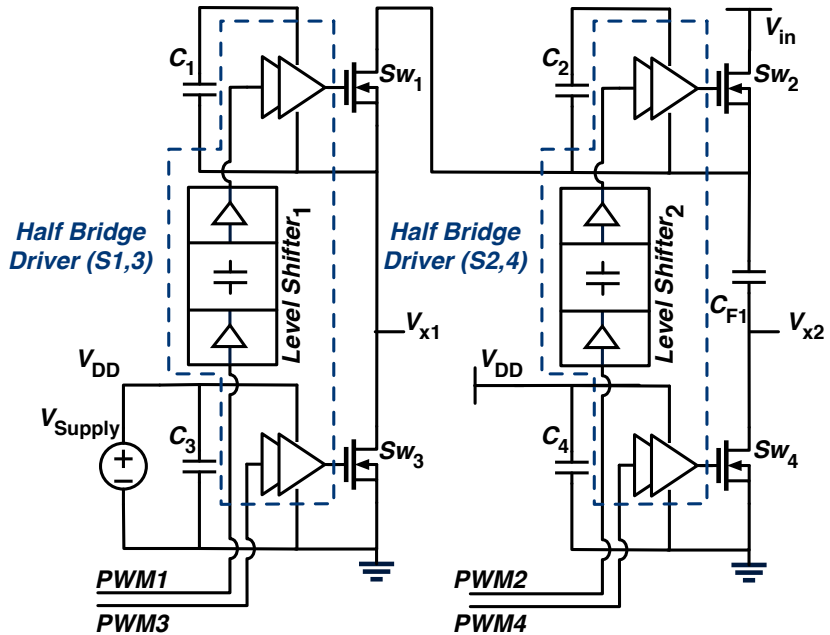

Fig. 2: Optimal use of half bridge drivers with integrated signal isolators.

half-bridge switch pairs, they may not immediately appear to be controllable by a single half-bridge driver IC. However, the power-transfer capacitor $\mathrm{C}_{\mathrm{F} 1}$ connecting them acts as a DC voltage source in operation transient, ensuring that their operation is logically complementary in the same manner of a half-bridge switch pair with a DC voltage separation equivalent to the voltage across $\mathrm{C}_{\mathrm{F} 1}, \mathrm{~V}_{C_{F 1}}$. Recognizing that the signal isolator built in a half-bridge driver IC is capable of this voltage separation, only one driver IC is utilized to drive $\mathrm{Sw}_{2}$ and $\mathrm{Sw}_{4}$ Particularly, the built-in signal isolator can bring the gate control signal up for $\mathrm{Sw}_{2}$ while the high-side driver for $\mathrm{Sw}_{2}$ can operate at a $\mathrm{V}_{C_{F 1}}$ from the ground. This proposed method results in more optimal utilization of gate driver IC and removes the need for an additional signal isolator that would have been needed for $\mathrm{Sw}_{2}$ in the traditional solution [23].

It is worth noting that the example circuits in Fig. 2 represents the lowest 2 levels of the SC stage in an N-level SCBC, where $\mathrm{N}$ can be a number greater than 2 [12], [14], [15]. This optimal use of half-bridge drivers can be extended to drive all other high-side switches in the stack in multi-level hybrid converters [10]-[12], [15], [26].

\section{POWER RAIL GENERATION}

Together with the new optimal half-bridge driver scheme presented in Section II, it is important to devise appropriate methods to power high-side gate drivers operated at different voltage domains. There are many methods to achieve this goal as also presented in the comprehensive review reported in [23]. However, the methods have their own limitations which come in the way of practical implementation for a compact and reliable solution. This paper investigates three suitable powering schemes for the multi-inductor hybrid converter and provide more detailed comparison and design insights to help converter designers choose a suitable solution.

Three powering schemes of particular interest are isolated power modules (Fig. 3a), regulated supplies from switch blocking voltage (Fig. 3b), and regulated supplies from cascaded bootstrap (Fig. 3c). In this work, they are 


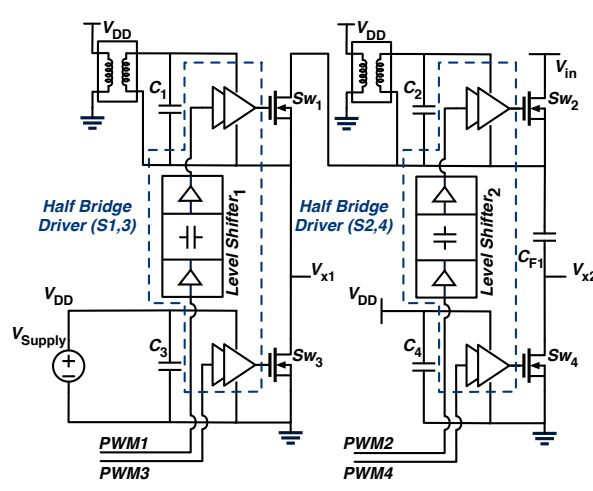

(a) Isolated modules

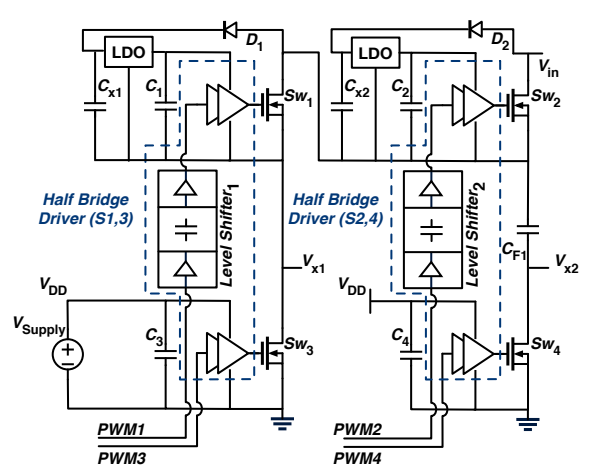

(b) Regulated supplies from switch blocking voltages

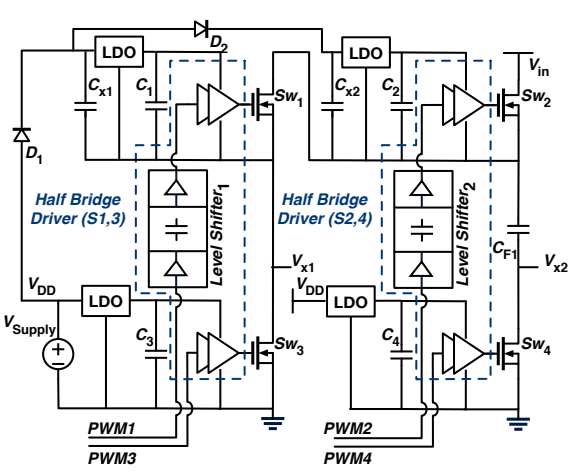

(c) Regulated supplies from cascaded bootstrap

Fig. 3: Optimal use of half-bridge drivers in multilevel hybrid converter with different powering schemes

implemented and demonstrated with the optimal half-bridge driver scheme described above. This section will provide descriptions of their operations, trade-offs, and design insights, while experimental demonstrations and measured performances are provided in Section IV.

\section{A. Use of isolated power modules}

As a straightforward method, isolated power modules can be used for driving high side switches, $\mathrm{Sw}_{1}$ and $\mathrm{Sw}_{2}$, shown in Fig. 3a. When the SCBC is extended to a larger number of levels, i.e. more SC stages, this powering method can be reliable as will be shown in Section IV. However, isolated power modules often have relatively large parasitic capacitance between the primary and secondary ground which can significantly limit the switching frequency and transient performance of the converters, and increase switching loss. Therefore, an exhaustive search for isolated DC-DC converter modules is recommended to find the lowest possible isolation capacitance to improve converter performance [27]. A key drawback of isolated power modules is their size that are often significantly larger than other components of the circuit, including both power switches and gate driver ICs [27], [28]. In addition, they are relatively inefficient, with efficiency limited to below $60 \%$, and capable of a relatively small load range [27]. The inefficiency increases gate driving losses that could be significant at light loads, which get worse when many modules are used for multiple high-side switches. It

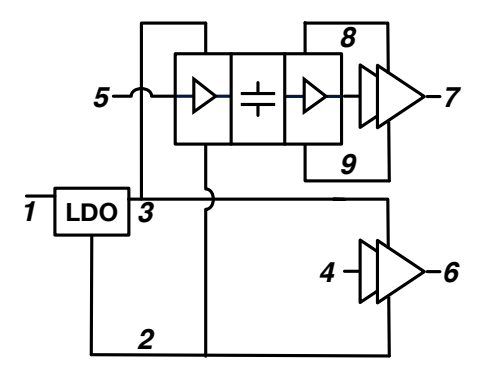

Fig. 4: Block diagram of LMG1210 [25], a half-bridge driver IC with integrated signal isolator and linear regulator (LDO), used in Fig. 5 is therefore desirable to find a more compact and efficient method to power high-side switch drivers.

\section{B. Regulated supplies from switch blocking voltages}

In multi-level converters, there are many intermediate voltage nodes switching at levels that can be utilized to generate flying voltage domains to drive high-side switches. From this recognition, another method to generate flying power rails from the converter switching nodes is explored to replace the isolated power modules in Fig. 3a. In the circuit shown in Fig. 3b, the blocking voltages of high-side switches $\mathrm{Sw}_{1}$ and $\mathrm{Sw}_{2}$ when they are off can be used to generate its $\mathrm{V}_{\mathrm{GS}}$ driving voltage. For example, when switch $\mathrm{Sw}_{1}$ is off, the voltage between its drain and source terminals can be used to charge capacitor $\mathrm{C}_{\mathrm{x} 1}$ via diode $\mathrm{D}_{1}$. In multilevel converters, this capacitor voltage is often larger and can then be regulated down to the required $\sim 5-\mathrm{V}$ level to drive $\mathrm{Sw}_{1}$ using a linear low-dropout regulator (LDO). A similar operation can be observed for $\mathrm{Sw}_{2}$ and its related circuit. Although this method consists of multiple discrete components, including an LDO, a diode, and two capacitors, because they are compact, the implementation space for this regulated flying supply domain is still significantly smaller compared to a standard isolated module. Therefore, this flying rail powering method helps reduce the overall area for gate driving circuits.

A key limitation of this powering method is the intrinsic nature of the LDO: its efficiency is low when there is a big difference between the blocking voltage $\left(\sim V_{C_{x 1}}\right)$ and the required $\mathrm{V}_{\mathrm{GS}}$ level $(\sim 5 \mathrm{~V})$. Unfavorably for this method, the blocking voltage depends on the converter topology, input voltage, and output current. When the input voltage increases, the blocking voltage increases linearly, and hence, the LDO efficiency decreases and gate driver power loss increases. Although having less impact, higher output currents also cause efficiency degradation in the LDOs and gate drivers as a consequence of larger voltage ripples on the main flying capacitors, leading to larger difference between the blocking voltages of high-side switches $\left(\sim V_{C_{x 1}}\right)$ and the LDOs' output $(\sim 5 \mathrm{~V})$. 


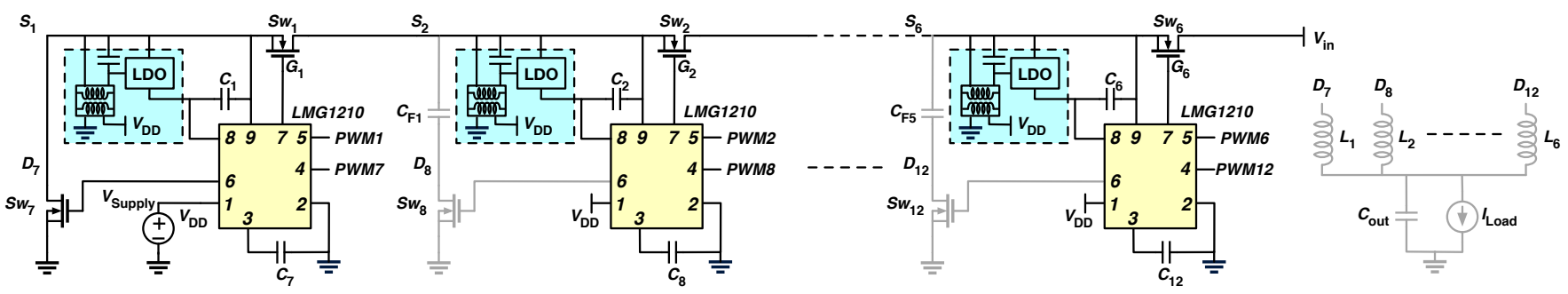

(a) 2-Phase 6-Inductor Hybrid Converter (2P6IHC) using optimized half-bridge drivers and isolated power supply modules.

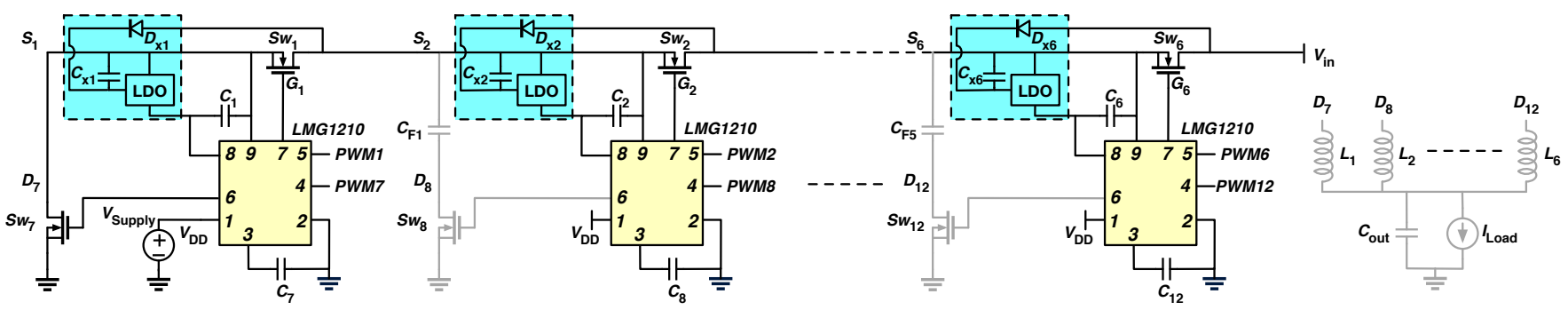

(b) 2-Phase 6-Inductor Hybrid Converter (2P6IHC) using optimized half-bridge drivers and regulated supplies from switch blocking voltages.

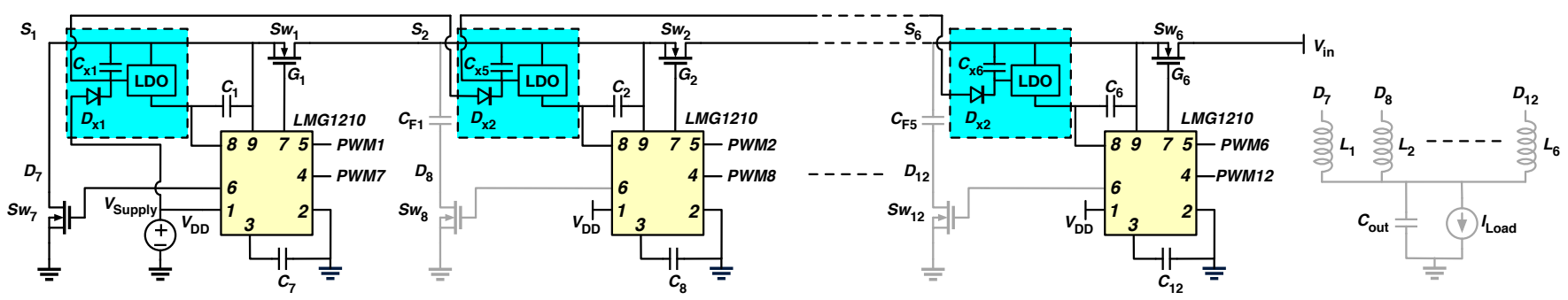

(c) 2-Phase 6-Inductor Hybrid Converter (2P6IHC) using optimized half-bridge drivers and regulated supplies from the cascaded bootstrap method.

Fig. 5: Schematic diagrams of the implemented converter prototype using different driver, signal isolator, and powering schemes (Stacked Switches and their driver circuits are highlighted)

\section{Regulated supplies from cascaded bootstrap circuits}

To reduce the voltage difference between the input and output of gate driver LDOs and avoid the dependence on the converter's input voltage and output current, another powering scheme for high-side gate drivers is desirable. Figure $3 \mathrm{c}$ shows a circuit for a cascaded bootstrap circuit with additional LDOs added to all power domains of gate drivers. Although this cascaded bootstrap structure still has a diode voltage loss at every stack level, the input supply voltage $\mathrm{V}_{\text {Supply }}$ can be increased to overcome the series diode stack to support the top driver while the LDOs regulate the same safe driving voltage for all the gate drivers. Particularly, in the circuit shown in Fig. $3 \mathrm{c}, \mathrm{V}_{\text {Supply }}$ is provided at $\sim 5.6 \mathrm{~V}$, accounting for $0.5 \mathrm{~V}$ diode drop in 2 cascaded stages to provide $\sim 5.1 \mathrm{~V}$ at $\mathrm{C}_{\mathrm{x} 2}$. From $\mathrm{C}_{\mathrm{x} 2}$, the LDO can have a $100 \mathrm{mV}$ drop-out voltage margin to regulate a $5 \mathrm{~V}$ supply for the flying voltage domain driving $\mathrm{Sw}_{2}$.

In this method, cascading bootstrap circuits for a large number of stack switches will result in a large accumulation of multiple diode drops, requiring a large $\mathrm{V}_{\text {Supply }}$ for operation. A large $\mathrm{V}_{\text {Supply }}$, in turn, put the LDOs at at the bottom and lower voltage domains of the stack at low efficiencies because of large input/output voltage difference. Therefore, there have been new engineering efforts in both industry and academia to replace the bootstrap diodes with synchronous active devices in a more integrated approach [4], [29]. This can mitigate the requirement for high $\mathrm{V}_{\text {Supply }}$ and reduce the number of LDOs to improve gate driver efficiency. This paper focuses on commercially available parts for our developments, but it is still worth mentioning that ultimately, one would like to have a similar more integrated solution to achieve a more optimal power driving scheme which will need further investigations.

\section{Discussion on recently popular charge-pump methods}

Double charge-pump bootstrap circuit together with LDOs was proposed as an alternative [30] illustrated in Fig. 6. This method uses a lower-level driver to charge-pump higher bootstrap voltage instead of using a separate switch as in [31], [32]. The key idea in double charge-pump circuits is to utilize two cascaded charge-pump to double the bootstrap voltage to overcome diode voltage drops, then use an LDO to regulate the driver supply voltage to the desired $\sim 5 \mathrm{~V}$ level. This method has been popular in recent days, and there is also a variation of this circuit named gate driven charge pump claimed preferred option for hybrid converters [23].

While the operations of the double charge pump circuits can use the same $V_{\text {Supply }}$ level, they suffer from a serious practical issue that comes from the loading effect on drivers at lower 

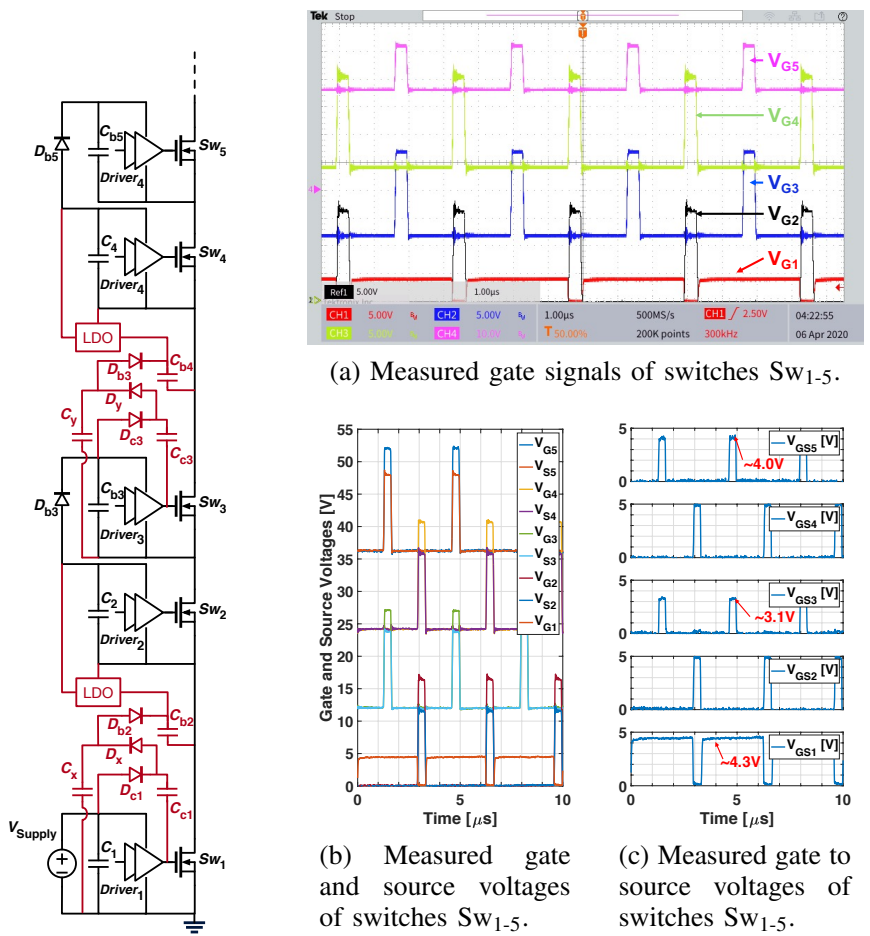

(a) Measured gate signals of switches $\mathrm{Sw}_{1-5}$.

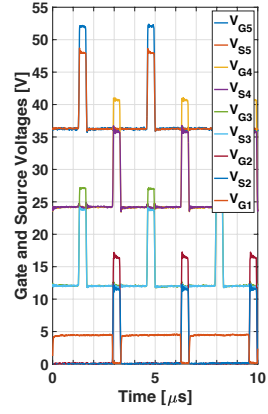

(b) Measured gate and source voltages of switches $\mathrm{Sw}_{1-5}$.

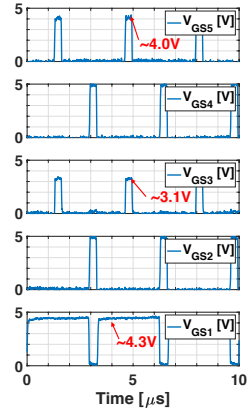

(c) Measured gate to source voltages of switches $\mathrm{Sw}_{1-5}$.
Fig. 6: Example of Fig. 7: Experimental waveforms of gate to charge-pumps source voltages of stacked switches using charge pump method

voltage domains. In today's gate driver ICs commercially available for GaN FETs, the drivers are generally designed to drive one switch. However, in the operations of double charge pump circuits, the bootstrapped driver $\left(\right.$ Driver $\left._{\mathrm{j}}\right)$ of each voltage domain needs to also drive the charge to bootstrap capacitors for higher domains. Particularly, during the charging of $\mathrm{C}_{\mathrm{x}}$ and $\mathrm{C}_{\mathrm{y}}$ in the double charge-pump circuit (Fig. 6), Driver $_{1}$ and Driver $_{3}$ carry turn-on current for switch $\mathrm{Sw}_{1}$ and switch $\mathrm{Sw}_{3}$ and the current required to charge capacitor $\mathrm{C}_{\mathrm{x}}$ and $\mathrm{C}_{\mathrm{y}}$ respectively. Furthermore, when more switches and stages are stacked on top of switch $\mathrm{Sw}_{4}$, the very heavy accumulated loading comes to Driver $_{3}$ and ultimately Driver $_{1}$. In practice, as gate driver is not generally designed to carry this level of large loads, its on resistance comes into the picture, lowering the actual $\mathrm{V}_{\mathrm{GS} 1}$ of $\mathrm{Sw}_{1}$ and $\mathrm{V}_{\mathrm{GS} 3}$ of $\mathrm{Sw}_{3}$. Because of these non-idealities, actual values of $\mathrm{V}_{\mathrm{GS} 1}$ and $\mathrm{V}_{\mathrm{GS} 3}$ can become as low as $\sim 4.3 \mathrm{~V}$ and $\sim 3.1 \mathrm{~V}$. This $\sim 3.1 \mathrm{~V}$ is far below the required level to properly turn on switch $\mathrm{Sw}_{3}$, leading to low converter efficiency and failure of $\mathrm{Sw}_{3}$. Note that, this effect is separate from the effect of the gate voltage drop from the bootstrap diode. In Fig. 6, bootstrap diode has effect on $V_{G S 3}$ and $V_{G S 5}$. The loading effect of the driver has effects on $\mathrm{V}_{\mathrm{GS} 1}$ and $\mathrm{V}_{\mathrm{GS} 3}$. As non-ideality of $\mathrm{V}_{\mathrm{GS} 3}$ comes from both the sources, the worst case can be seen on the magnitudes of it, and it has higher chances of failures. Depending on the nature of a multilevel converter, a failure of one switch can expose other switches to voltage levels much higher than their ratings, causing cascaded failures and irreversible damages to the whole converter. Experimental demonstrations of this phenomenon has been provided in Fig. 7. The circuit in Fig. 6 has been implemented, all the gate and source voltages have been measured, and the gate to source voltages have been calculated from the measurements.

This practical problem is common in many double chargepump circuits. The gate-driven charge pump method claimed as preferred in [23] also suffers from the same problem. The key solution to this problem is to construct the converter's power switches of the converter, rather than the gate drivers, to drive the charge-pump capacitors, as shown in the two powering schemes discussed in subsection III-B (Fig. 3b) and Section III-C (Fig. 3c) above. The strength of the main power switches is capable of satisfying the current stress needed by multiple gate driver bootstrap circuits.

\section{HARDWARE IMPLEMENTATIONS AND EXPERIMENTAL RESULTS}

In order to verify their steady-state and transient operations and compare their trade-offs, the gate driving and powering methods discussed above were implemented and re-configurable in a multilevel multi-inductor hybrid (MIH) converter, whose schematic is in Fig. 1, detailed schematics in Fig. 5, and the hardware in Fig. 8. To simplify the drawing of overall converter schematics and connections, Fig. 5 uses a 9-pin block for the the LMG1210 gate driver IC that has an integrated signal isolator, while the more detailed diagram of LMG1210 is shown in Fig. 4.

The hybrid converter is operated with 2 energizing phases and 6 inductors (2P6IHC). The 2P6IHC prototype was implemented with the isolator-less, optimized half-bridge driver method described in Section II, and can be reconfigured to support three different high-side driver powering schemes: isolated power supply modules (Fig. 5a), regulated supplies from switch blocking voltages (Fig. 5b), and regulated supplies from cascaded bootstrap method (Fig. 5c). The converter has twelve switches in total, six of which, $\mathrm{Sw}_{7-12}$, are at the

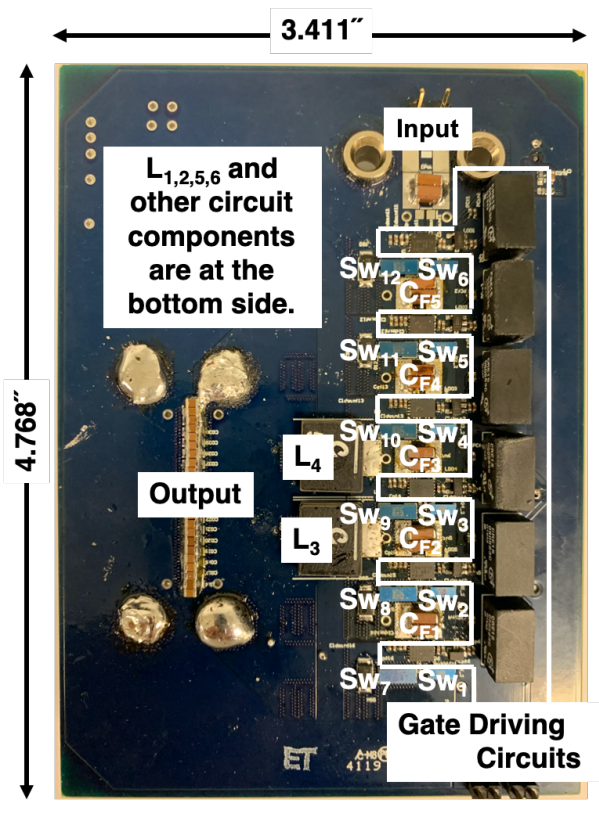

Fig. 8: Multi-level multi inductor hybrid converter prototype, reconfigurable for different schemes in gate driver, signal isolator, and powering flying voltage domains. 


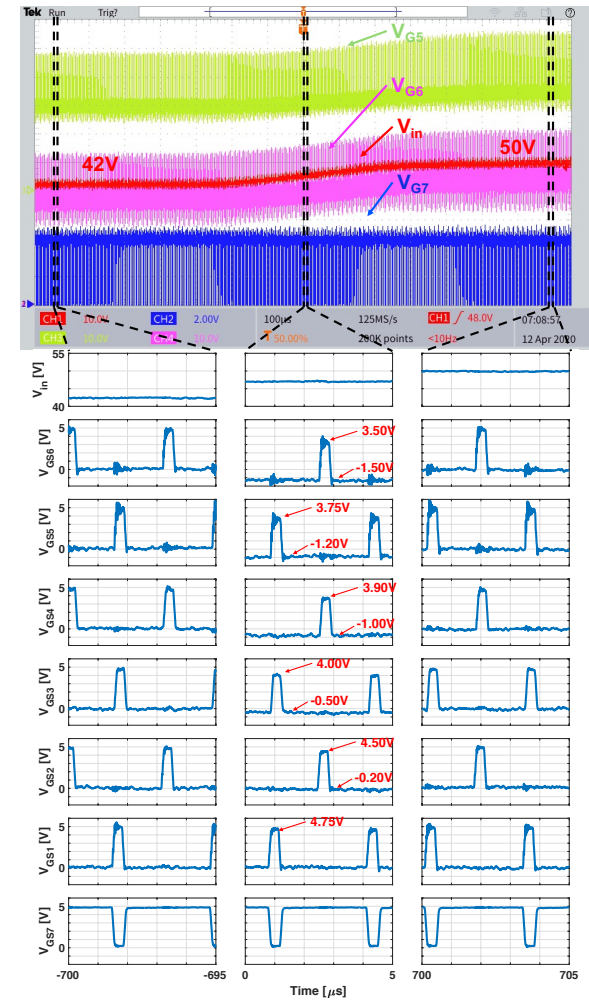

(a) $42 \mathrm{~V}$ to $50 \mathrm{~V}$ line transient waveforms.

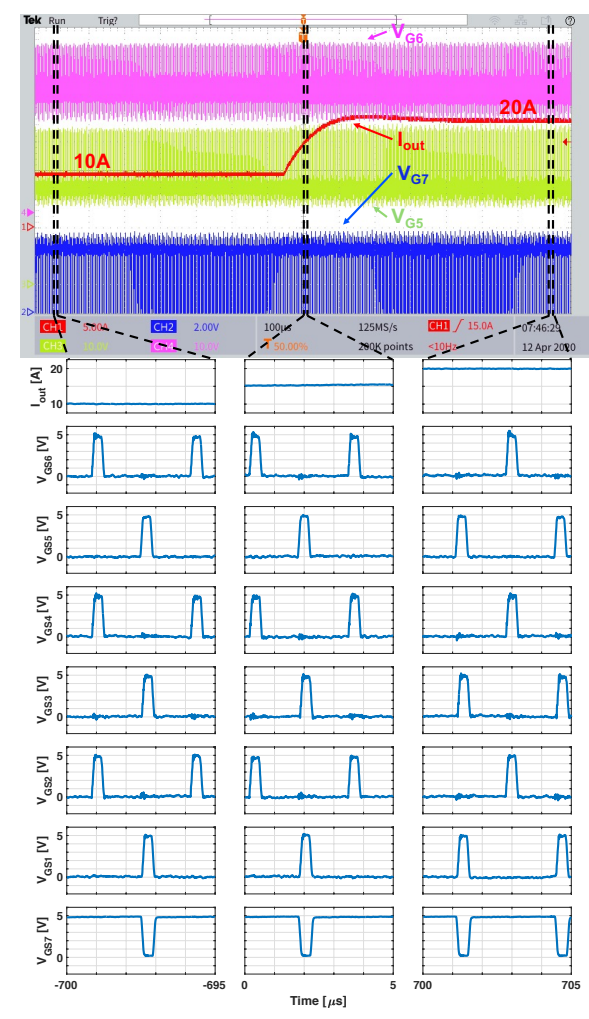

(b) $10 \mathrm{~A}$ to $20 \mathrm{~A}$ load transient performance.

Fig. 9: Measured transient gate signals of the $2 \mathrm{P} 6 \mathrm{IHC}$ prototype using optimized half-bridge drivers and isolated power modules.

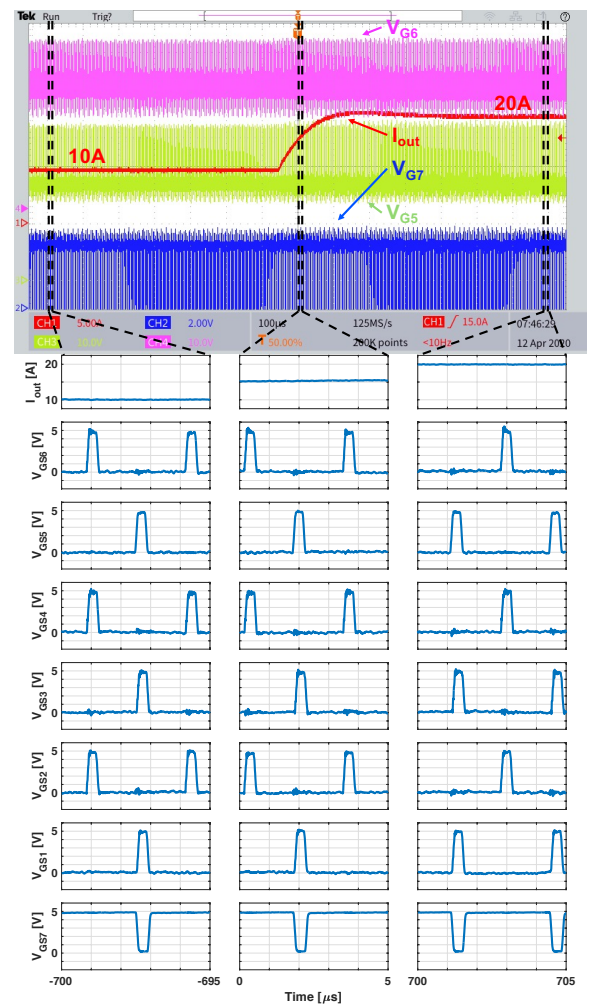

(a) $42 \mathrm{~V}$ to $50 \mathrm{~V}$ line transient waveforms.

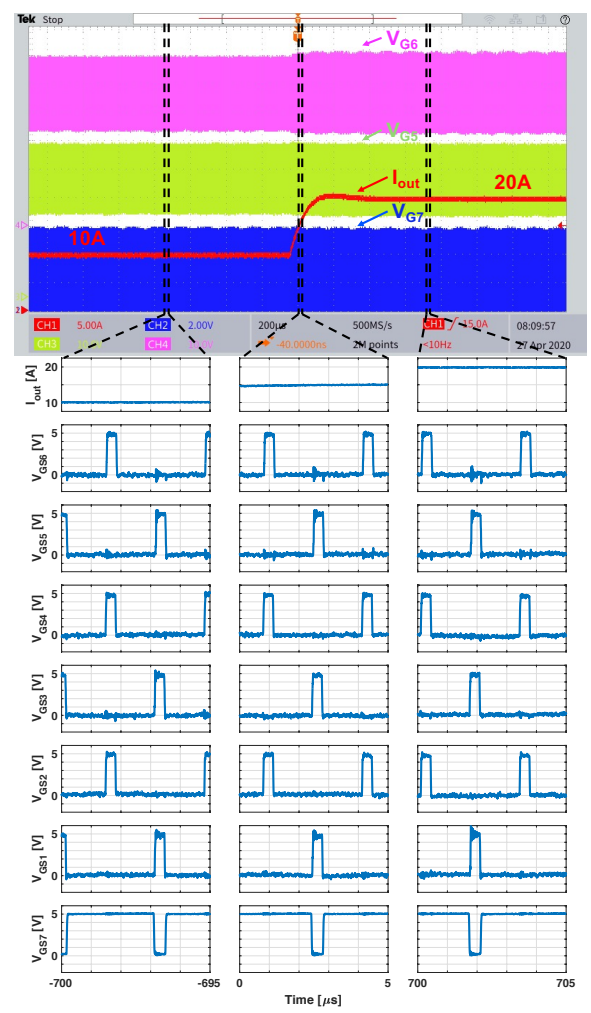

(b) $10 \mathrm{~A}$ to $20 \mathrm{~A}$ load transient performance.

Fig. 10: Measured transient gate Fig. 11: Measured transient gate signals signals of the $2 \mathrm{P} 6 \mathrm{IHC}$ prototype using of the $2 \mathrm{P} 6 \mathrm{IHC}$ prototype using optimized the optimized half-bridge driver and half-bridge driver and regulated supplies regulated supplies from switch blocking from cascaded bootstrap method. voltages.

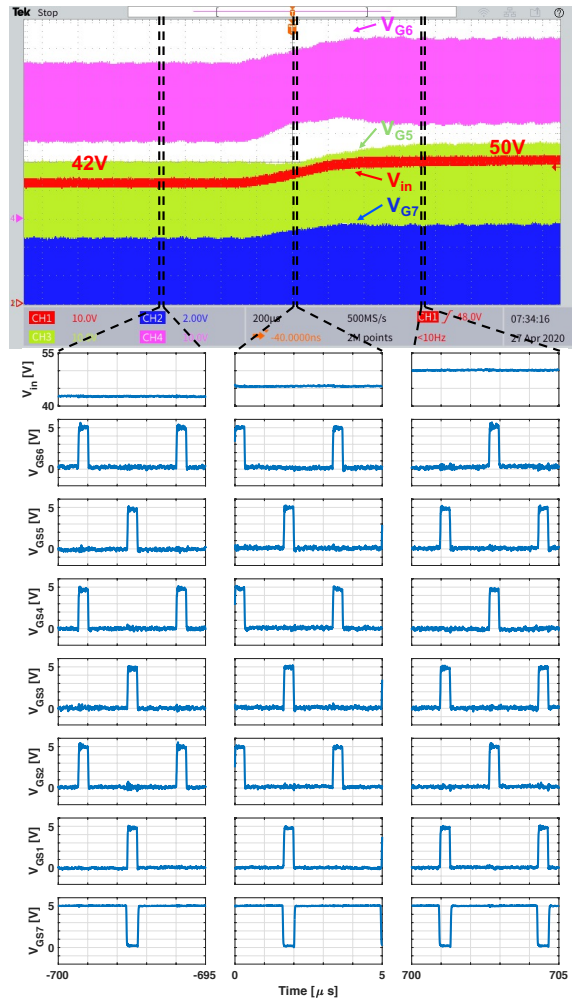

(a) $42 \mathrm{~V}$ to $50 \mathrm{~V}$ line transient waveforms.

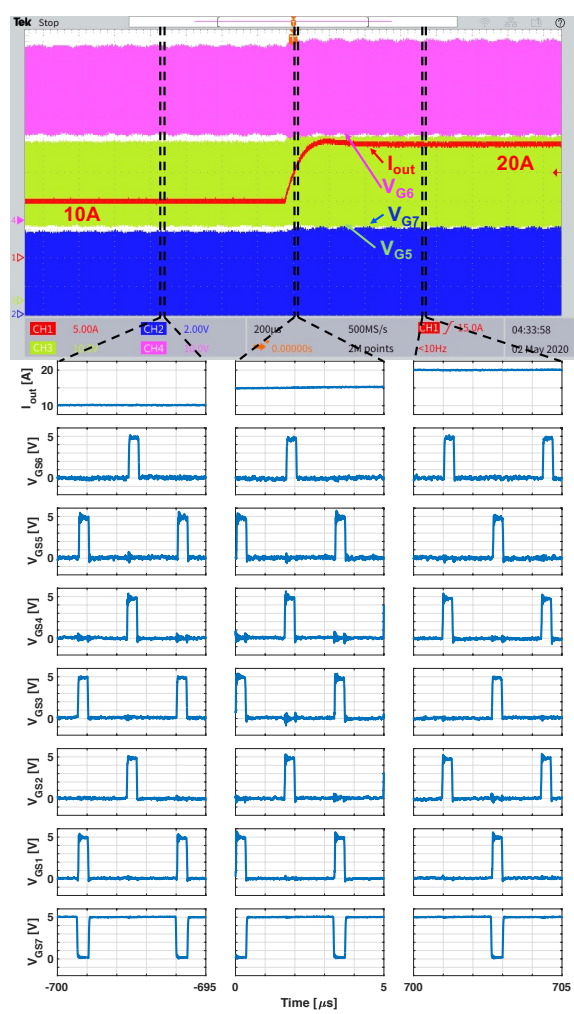

(b) $10 \mathrm{~A}$ to $20 \mathrm{~A}$ load transient performance. 
TABLE I: List of components and comparison for different gate drivers and powering schemes

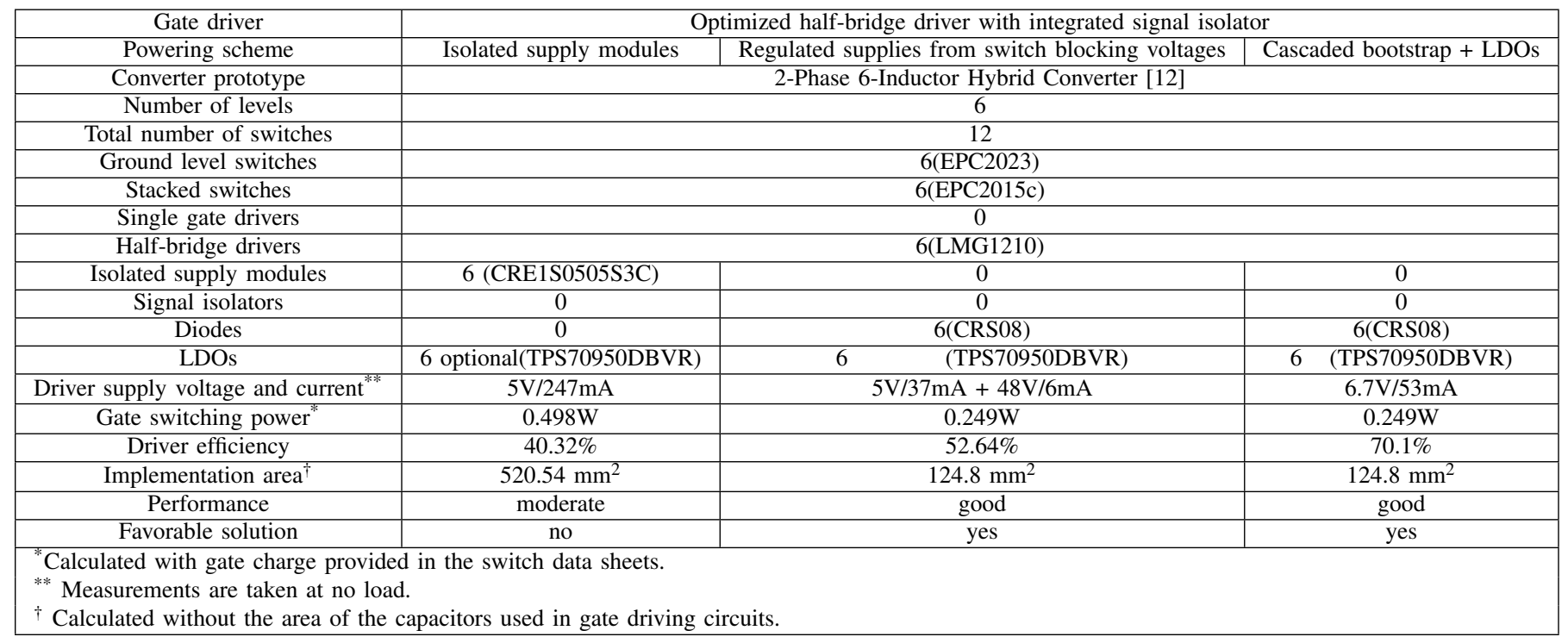

ground level and the other six, $\mathrm{Sw}_{1-6}$, are stacked on $\mathrm{Sw}_{7}$. Six half-bridge drivers LMG1210 ICs are employed to drive the 12 switches in this implementation. While $\mathrm{Sw}_{1}-\mathrm{Sw}_{7}$ pair is directly connected and operated as a conventional halfbridge, the remaining 5 switch pairs, $\mathrm{Sw}_{2}-\mathrm{Sw}_{8}$ to $\mathrm{Sw}_{6}-\mathrm{Sw}_{12}$, are connected via 5 flying capacitors, $\mathrm{C}_{\mathrm{F} 1}$ to $\mathrm{C}_{\mathrm{F} 5}$, respectively. Note that the LMG1210 driver conveniently comes with an integrated LDO for its bottom driver. Therefore, no additional discrete $\mathrm{LDO}$ is needed for the ground switches $\mathrm{Sw}_{7}-\mathrm{Sw}_{12}$.

In the circuit in Fig. 5a, an LDO is used after each isolated power module to regulate the gate driver supply voltage at 25.0-5.5 V, recommended for sensitive GaN switches [27], [33]. These LDOs can be avoided if the isolated power modules can maintain a tight voltage close to $5 \mathrm{~V}$. Similarly, Fig. 5b and Fig. 5c shows the schematic of the 2P6IHC prototype employing regulated supplies from switch blocking voltages and from the cascaded bootstrap method for stacked gate drivers, respectively.

The 2P6IHC prototype with these three driver powering schemes was also tested for reliability in $8 \mathrm{~V}$ line transients and 10A load transients as shown in Figs. 9, 10, and 11. In these figures, all the gate and source are measured. Gate to source voltages are calculated from these measurements and plotted before, during and after the transients. Their steady-state operations before and after the transients show the intended operations of the discussed power rail generation methods to provide the right $5 \mathrm{~V}_{\mathrm{GS}}$ for all stacked $\mathrm{GaN}$ switches.

Looking closely during the line transients in Fig. 9a reveals that, the parasitic capacitance of the isolated modules have come into effect to cause slow changes in the converter switching levels, as well as the voltages of the power switches's sources and gates. As a result, $\mathrm{V}_{\mathrm{GS}}$ of some of the high side switches are reduced in line transients. This phenomenon exposes the switches very close to failure and thus, can be problematic in practical implementations. This problem can be worse with faster line transients. Other two methods using switch blocking voltages and cascaded bootstrap powering schemes do not suffer from this problem.
It can be seen that the gate and source voltages maintain at the expected level to maintain $\mathrm{V}_{\mathrm{GS}}$ close to $5 \mathrm{~V}$ throughout the line transients in Fig. 10a and 11a.

Fig. 9b, 10b and $11 \mathrm{~b}$ also lists the load transient performance of the discussed power rail generation schemes. There is no significant difference among the schemes for the load transient performances.

All these results validate the expected functionality, stable and reliable operations of both switch blocking voltages and cascaded bootstrap powering schemes under both load and line transient stresses, proving their worthiness in applications. Isolated modules can also be a good straight-forward simple candidate if the the application does not require the converter to go through fast line transients.

Note that, the powering method where switch blocking voltages are used to derive the power rails does not work when the input voltage is low such that switch blocking voltages are reduced to less than $5 \mathrm{~V}$, i.e. not enough voltage to provide to the LDOs. This scenario can be seen at startup when input voltage starts increasing from zero. In this particular implementation, this powering scheme starts working for an input voltage from $\sim 18 \mathrm{~V}$. In normal operations of the converter with $\mathrm{V}_{\text {in }}$ at $40-60 \mathrm{~V}$, switch blocking voltages are at least $\sim 6.5 \mathrm{~V}$ for LDOs for $\mathrm{Sw}_{1,6}$ and $\sim 13 \mathrm{~V}$ for LDOs for $\mathrm{Sw}_{2-5}$. These voltages are high enough to warrant the intended operation of the circuits.

Table I includes a comparison of the gate driving and powering methods investigated in this paper on a number of characteristics and trade-offs. Optimal utilization of the signal isolator built-in to commercial half-bridge drivers creates new opportunities to save significant areas of discrete signal isolators. This method combined with either regulated power supplies from switch blocking voltages or regulated power supplies with cascaded bootstrap can yield an overall significantly smaller area while achieving both high driving efficiency and reliability. In these better powering schemes, the cascaded bootstrap and LDO regulation for every stage produce a high driving efficiency of $\sim 70 \%$ because of the 
flexibility to set small voltage drops across the LDOs. The driving efficiency is calculated as

Note that, although the converter prototype is shown to operate with 2 energizing phases in this paper, these gate driver and powering schemes also work in other operation modes with more energizing phases, such as the 6-phase operation shown in [34].

\section{CONCLUSION}

In summary, a number of methods to power and drive stacked switches in a GaN-based multi-level multiinductor (MIH) hybrid converter have been discussed and experimentally demonstrated in this work. Their trade-offs and challenges have been discussed from different aspects of practical implementations to help designers choose a suitable solution for particular design needs. In practice, gate driver circuits can cause faults and failures in converters using sensitive GaN FETs. Hence, it is desirable to choose a set of proper level shifting techniques for PWM signals and powering schemes for reliable operations while maintaining an overall compact and efficient implementation. An isolator-less method has been devised for small area implementation and demonstrated with different powering schemes for elevated domains. A 6-level 6-inductor hybrid converter prototype was used to demonstrate the gate driving and powering schemes, validate their operations, and discuss performance and design trade-offs. The experiments, circuits, and discussions in this paper are aimed to be a good source of reference for future engineering efforts in designing multi-level hybrid converters.

\section{REFERENCES}

[1] J. Zhu and D. Maksimović, "A Family of Transformerless Stacked Active Bridge Converters," in 2019 IEEE Applied Power Electronics Conference and Exposition (APEC), Mar. 2019, pp. 19-24.

[2] S. Webb, T. Liu, and Y.-F. Liu, "A Two-Phase Zero-Inductor Voltage Converter for Datacenter and Server Applications," in 2019 IEEE Applied Power Electronics Conference and Exposition (APEC), Mar. 2019, pp. 2255-2262.

[3] S. Khatua, D. Kastha, and S. Kapat, "Novel Transformer-Less DAB Converters for the Regulated First-Stage of a Two-Stage 48 V VRM," in 2018 IEEE Energy Conversion Congress and Exposition (ECCE), Sep. 2018, pp. 5258-5264.

[4] S. Biswas and D. Reusch, "GaN Based Switched Capacitor ThreeLevel Buck Converter with Cascaded Synchronous Bootstrap Gate Drive Scheme," in 2018 IEEE Energy Conversion Congress and Exposition (ECCE), Sep. 2018, pp. 3490-3496.

[5] S. Khatua, D. Kastha, and S. Kapat, "A New Single-Stage 48-V-Input VRM Topology Using an Isolated Stacked Half-Bridge Converter," IEEE Transactions on Power Electronics, vol. 35, no. 11, pp. 11976-11987, Nov. 2020.

[6] A. Kumar, S. Pervaiz, and K. K. Afridi, "Single-stage isolated 48Vto-1.8V point-of-load converter utilizing an impedance control network and integrated magnetic structures." IEEE, Jul. 2017, pp. 1-7.

[7] J. S. Rentmeister and J. T. Stauth, "A 48V:2V flying capacitor multilevel converter using current-limit control for flying capacitor balance," in 2017 IEEE Applied Power Electronics Conference and Exposition (APEC), Mar. 2017, pp. 367-372.

[8] P. Jain, A. Prodić, and A. Gerfer, "Wide-input high power density flexible converter topology for dc-dc applications," in 2016 IEEE Applied Power Electronics Conference and Exposition (APEC), Mar. 2016, pp. 2553-2560.

[9] S. Khatua, D. Kastha, and S. Kapat, "A Non-Isolated Single-Stage 48Vto-1V VRM with a Light Load Efficiency Improvement Technique," in 2018 IEEE Energy Conversion Congress and Exposition (ECCE), Sep. 2018, pp. 143-148.
[10] G.-S. Seo, R. Das, and H.-P. Le, "Dual Inductor Hybrid Converter for Point-of-Load Voltage Regulator Modules," IEEE Transactions on Industry Applications, vol. 56, no. 1, pp. 367-377, Jan. 2020.

[11] R. Das, G. Seo, D. Maksimovic, and H.-P. Le, "An 80-W 94.6\%-Efficient Multi-Phase Multi-Inductor Hybrid Converter," in 2019 IEEE Applied Power Electronics Conference and Exposition (APEC), Mar. 2019, pp. 25-29.

[12] R. Das and H.-P. Le, "A Regulated 48V-to-1V/100A 90.9\%Efficient Hybrid Converter for POL Applications in Data Centers and Telecommunication Systems," in 2019 IEEE Applied Power Electronics Conference and Exposition (APEC), Mar. 2019, pp. 1997-2001.

[13] O. Kirshenboim and M. M. Peretz, "High-Efficiency Nonisolated Converter With Very High Step-Down Conversion Ratio," IEEE Transactions on Power Electronics, vol. 32, no. 5, pp. 3683-3690, May 2017.

[14] K. Matsumoto, K. Nishijima, T. Sato, and T. Nabeshima, "A twophase high step down coupled-inductor converter for next generation low voltage CPU," in 8th International Conference on Power Electronics ECCE Asia, May 2011, pp. 2813-2818.

[15] K. Abe, K. Nishijima, K. Harada, T. Nakano, T. Nabeshima, and T. Sato, "A Novel Three-Phase Buck Converter with Bootstrap Driver Circuit," in 2007 IEEE Power Electronics Specialists Conference, Jun. 2007, pp. 1864-1871.

[16] V. Yousefzadeh, E. Alarcon, and D. Maksimovic, "Three-level buck converter for envelope tracking applications," IEEE Transactions on Power Electronics, vol. 21, no. 2, pp. 549-552, Mar. 2006.

[17] Y. Liu, A. Kumar, D. Maksimovic, and K. K. Afridi, "A High-PowerDensity High-Efficiency Three-Level Buck Converter for Cellphone Battery Charging Applications," in 2018 IEEE Energy Conversion Congress and Exposition (ECCE), Sep. 2018, pp. 5265-5270.

[18] P. S. Shenoy, M. Amaro, J. Morroni, and D. Freeman, "Comparison of a Buck Converter and a Series Capacitor Buck Converter for High-Frequency, High-Conversion-Ratio Voltage Regulators," IEEE Transactions on Power Electronics, vol. 31, no. 10, pp. 7006-7015, Oct. 2016.

[19] J. Zhu, R. Schuess, and D. Maksimovic, "General Properties and Synthesis of Transformerless Stacked Active Bridge Converters," in 2019 20th Workshop on Control and Modeling for Power Electronics (COMPEL), Jun. 2019, pp. 1-6.

[20] L. Zhang and S. Chakraborty, "An Interleaved Series-Capacitor Tapped Buck Converter for High Step-Down DC/DC Application," IEEE Transactions on Power Electronics, vol. 34, no. 7, pp. 6565-6574, Jul. 2019.

[21] "Si8410/20/21 (5 kV) Si8422/23 (2.5 \& 5 kV) Data Sheet," Silicon Laboratories, Inc., Tech. Rep.

[22] “3.0 kV rms, Dual-Channel Digital Isolator ADuM120N/ADuM121N," Analog Devices, Inc., Tech. Rep.

[23] Z. Ye, Y. Lei, W.-C. Liu, P. S. Shenoy, and R. C. N. Pilawa-Podgurski, "Improved Bootstrap Methods for Powering Floating Gate Drivers of Flying Capacitor Multilevel Converters and Hybrid Switched-Capacitor Converters," IEEE Transactions on Power Electronics, vol. 35, no. 6, pp. 5965-5977, Jun. 2020.

[24] A. Kumar and K. K. Afridi, "Single-stage isolated 48V-to-1.8V pointof-load converter utilizing an impedance control network for wide input range operation," in 2017 IEEE Energy Conversion Congress and Exposition (ECCE), Oct. 2017, pp. 2003-2009.

[25] "LMG1210 200-V, 1.5-A, 3-A half-bridge MOSFET and GaN FET driver with adjustable dead time for applications up to $50 \mathrm{MHz}$," Texas Instruments Inc., Tech. Rep.

[26] L. Pham-Nguyen, V.-Q. Nguyen, D.-M. Nguyen, H.-D. Han, K.H. Nguyen, and H.-P. Le, "A 14-W 94\%-Efficient Hybrid DC-DC Converter with Advanced Bootstrap Gate Drivers for Smart Home LED Applications," in 2018 IEEE Energy Conversion Congress and Exposition (ECCE), Sep. 2018, pp. 4744-4749.

[27] "CRE1 Series:Isolated 1W Single Output Isolated DC-DC converters," Murata Power Solutions Inc., Tech. Rep.

[28] “DC/DC Converter,” Recom Power, Tech. Rep.

[29] "EPC2107 - Enhancement-Mode GaN Power Transistor Half-Bridge with Integrated Synchronous Bootstrap," Efficient Power Corporation, Tech. Rep.

[30] Z. Ye, Y. Lei, W.-c. Liu, P. S. Shenoy, and R. C. N. PilawaPodgurski, "Design and implementation of a low-cost and compact floating gate drive power circuit for GaN-based flying capacitor multilevel converters." IEEE, Mar. 2017, pp. 2925-2931.

[31] S. Park and T. Jahns, "A self-boost charge pump topology for a gate drive high-side power supply," IEEE Transactions on Power Electronics, vol. 20, no. 2, pp. 300-307, Mar. 2005. 
[32] M. Kovacic and S. Krishnamurthy, "Gate drive power supply for multilevel converter," US Patent US20 150318 788A1, Nov., 2015.

[33] A. Lidow and J. Strydom, "eGaN® FET Drivers and Layout Considerations," Efficient Power Corporation, Tech. Rep., 2016.

[34] T. Xie, R. Das, G. Seo, D. Maksimovic, and H.-P. Le, "Multiphase Control for Robust and Complete Soft-charging Operation of Dual Inductor Hybrid Converter," in 2019 IEEE Applied Power Electronics Conference and Exposition (APEC), Mar. 2019, pp. 1-5.

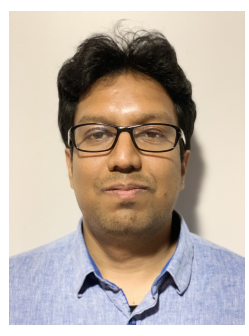

Ratul Das (S'15) received the B.Sc. and M.Sc. degrees in Electrical and Electronic Engineering from the Bangladesh University of Engineering and Technology (BUET), Dhaka, Bangladesh, in 2015 and 2017, respectively. He worked in the Integrated Power Electronics and Energy Efficient Systems (iPower3Es) group at the Colorado Power Electronics Center (CoPEC), University of Colorado at Boulder from 2017 to 2019 as a graduate student researcher. Currently, he is focusing on his journey towards the PhD degree with the iPower3Es group at the University of California San Diego, La Jolla, CA. Mr. Das was the recipient of a Gold Outstanding Graduate Student Researcher Award 2019 from the ECEE department of University of Colorado Boulder, a Best Paper Award at IEEE COMPEL 2019 and a second prize award in IEEE ECCE 2019 student project demonstrations.

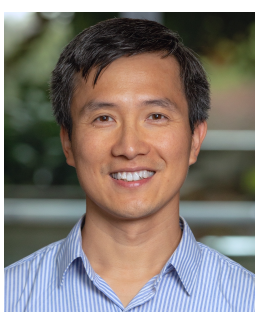

Hanh-Phuc Le (M'13-SM'19) received the B.S. degree from the Hanoi University of Science and Technology (HUST), Hanoi, Vietnam, in 2004, the M.S. degree from the Korea Advanced Institute of Science and Technology (KAIST), Daejeon, South Korea, in 2006, and the $\mathrm{PhD}$ degree from the University of California at Berkeley, Berkeley, CA, USA, in 2013, all in Electrical Engineering. He is currently an Assistant Professor at the Electrical and Computer Engineering Department, the University of California at San Diego (UCSD), La Jolla, CA, USA. He was also with the University of Colorado at Boulder, Boulder, CO, USA, from 2016 to 2019. Dr. Le was a recipient of the 2012-2013 IEEE SSCS PreDoctoral Achievement Award, and the 2013 Sevin Rosen Funds Award for Innovation at University of California at Berkeley. He is currently the Chair of the Technical Committee on Power Conversion Systems and Components in the IEEE Power Electronics Society (IEEE PELS TC2). 